\title{
DBS in Dystonia and Other Hyperkinetic Movement Disorders
}

\author{
A. Barbey, MD \\ J. Bloch, MD \\ F. J. G. Vingerhoets, $M D^{*}$
}

Address

"Department of Neurology, Centre Hospitalier Universitaire Vaudois (CHUV), Rue de Bugnon 21, CH-1011, Lausanne, Switzerland

Email: Francois.Vingerhoets@chuv.ch

Published online: 11 August 2015

(C) Springer Science+Business Media New York 2015

This article is part of the Topical Collection on Movement Disorders

Keywords Hyperkinetic movement disorders - Deep brain stimulation (DBS) - Dystonia - Tremor - Tardive dyskinesia • Gilles de la Tourette syndrome $\cdot$ Chorea $\cdot$ Ballism

\section{Opinion statement}

The diagnosis and appropriate treatment of hyperkinetic movement disorders require a work up of potentially reversible metabolic, infectious and structural disorders as well as side effects of current medication. In pharmacoresistant movement disorders with a disabling impact on quality of life, deep brain stimulation (DBS) should be considered. At different targets, DBS has become an established therapy for Parkinson's disease (GPiSTN), tremor (VIM) and primary dystonia (GPi) with reasonable perioperative risks and side effects, established guidelines and some clinical and radiological predictive factors. In contrast, for other hyperkinetic movement disorders, including secondary dystonia, Gilles de la Tourette, chorea and ballism, only few data are available. Definite targets are not well defined, and reported results are of less magnitude than those of the recognized indications. In this expanding therapeutical field without worked out recommendations, an individual approach is needed with DBS indication assessment only after rigorous multidisciplinary scrutiny, restricted to expert centres.

\section{Introduction}

Hyperkinetic movement disorder is a heterogeneous group, including tremor, dystonia, chorea, myoclonus, tics, stereotypies, restless legs syndrome and various other disorders. Differential diagnosis of hyperkinetic movement disorders is very broad. For better pathophysiological understanding and management, they can be classified in primary and secondary movement disorders, the latter due to heredodegenerative diseases or secondary to known causes. Evaluation contains at first exclusion of potentially reversible causes. 
After extensive laboratory investigation, workup includes always a neuroimaging (MRI) to detect possible ischemia, haemorrhage or neoplasia. Pharmacological treatment is often limited by side effects, making deep brain stimulation (DBS) an interesting alternative approach. First attempts of functional surgery as additional treatment to medical management started in the early 50s, with lesions of various anatomical targets. Preponderant limiting factors were the occurrence of irreversible side effects, particularly in bilateral approaches. In the late 1980s, Benabid et al. reported that high frequency DBS was mimicking lesion for treatment of tremor [1]. Thereafter, DBS has been shown to be as efficacious as the thalamotomy but with lesser adverse effects thanks to the advantage of adjustable stimulation parameters [2]. Since then, the potential indications of DBS have been explored in multiple fields of neurology and psychiatry. Although DBS mechanisms of action are not fully understood, it is thought to modulate the expression of dysfunctional neuronal networks. Potential risk of DBS surgery includes haemorrhage (1.9\%), infection (4.7\%), electrode breakage $(0.94 \%)$ and lead migration or misplacement $(0.94 \%)$ with an overall mortality of $0.94 \%$ [3]. Overall, serious side effects of DBS are rare, with little impact on cognitive function and behaviour $[4 \bullet \bullet]$.
Postoperative side effects after chronic stimulation such as muscular contractions or visual flashes, the latter due to proximity to optical tract, are mostly mild and reversible by adjusting the stimulation parameters [5]. The electrode implantation is preferably performed under local anaesthesia, as optimizing of electrode placing and consequently its therapeutical efficacy and potential side effects can be tested preoperatively. Stereotactic neurosurgery with DBS has become an established therapy of selected movement disorders and revolutionized treatment especially in Parkinson's disease, essential tremor and dystonia after its approval by the US Food and Drug Administration in 1997 (essential tremor (ET)), respectively, in 2002 (for Parkinson's disease (PD) and dystonia) and is also used in psychiatric disorders as major depression and obsessive-compulsive disorder. An increasing number of studies suggest its efficacy in treatment of secondary dystonia and in chorea, Gilles de la Tourette's syndrome, tardive dyskinesia and ballism, turning DBS in an interesting alternative therapeutical approach, for all hyperkinetic movement disorders.

We will focus on actual state of knowledge and guidelines of DBS in following hyperkinetic movement disorders: dystonia, tardive dyskinesia, tremor, Gilles de la Tourette's syndrome, chorea and ballism.

\section{Treatment}

\section{DBS and dystonia}

Dystonia is a movement disorder characterized by sustained or intermittent agonist and antagonist muscles co-contractions, causing abnormal posture and/ or movements. Dystonia may be triggered or worsened by voluntary action and associated with overflow muscle activation; sensory tricks with "gestes antagonists" can alleviate them. Their division in primary and secondary dystonia on the basis of their aetiology is essential for pathophysiological understanding and appropriate management. Further classification according to their localization (focal, segmental, multifocal, generalized, hemidystonia) and age at onset (infancy, childhood, adolescence, adulthood) can be used.

Current EFNS guidelines (2011) for diagnosis and treatment of dystonia recommend the use of DBS in primary dystonia forms, in particular primary segmental and generalized dystonia, as well as in tardive dystonia, when

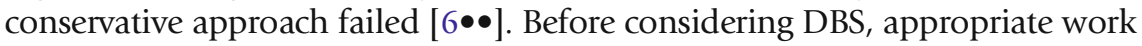
up, including MRI, is mandatory to differentiate primary and secondary dystonia $[6 \bullet \bullet$. By analogy to other indications, resistance to at least two different classes of medications at highest supportable dosages should be documented. Deep brain stimulation of internal globus pallidus (GPi) is considered as an 
established dystonia treatment for refractory primary segmental and generalized dystonia (level A evidence) with significant improvement of quality of life starting after 3-6 months $[6 \bullet \bullet, 7,8 \bullet \bullet]$. GPi is the main output of basal ganglia network. GPi-DBS influences the direct and the indirect pathways that are postulated to dysfunction in dystonia [9]. Recording oscillatory activity through implanted electrodes supports this approach, given that oscillatory theta activity is elevated in $\mathrm{GPi}$, coherent to phasic dystonic muscle contraction [9].

Posteroventral GPi stimulation has the best overall effect and is superior for the arm and the trunk, whereas anterodorsal stimulation shows similar efficacy for the leg [10]. In generalised and segmental dystonia, two multicentre controlled studies showed a good risk/benefit ratio for GPi-DBS, with mean improvements of the dystonia motor score of 51 and $42 \%[11,12]$. Clinical response to DBS in dystonia is typically gradually increasing over several weeks to months, and its efficacy is sustained [7] in primary dystonia up to 8 to 10 years in DT1-positive patients $[4 \bullet \bullet, 13,14]$. However, only a poor or no response may occur, even in patients that are not different in clinical profile or electrode placement from those with good response $[15,16]$. Predictive factors of good postoperative outcome in primary dystonia are younger age at the time of surgery $(<21$ years old) and shorter duration of symptoms ( $<15$ years), while age at onset and

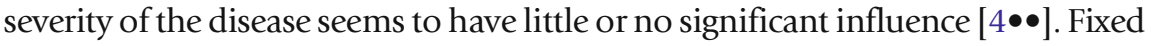
skeletal deformities are associated with poorer outcome [17]. In rare cases of cervical dystonia and blepharospasm, after several years of stimulation, symptoms did not reappear after cessation of DBS, suggesting that DBS could induce plastic changes $[4 \bullet \bullet]$. Overall, serious side effects of bilateral GPi-DBS are rare $[4 \bullet \bullet]$. Nevertheless, pre- and postoperatory neuropsychological and psychiatric assessment is recommended, given the high psychiatric comorbidity in the dystonia population and few reports of suicide after GPi-DBS implantation $[8 \bullet \bullet$. To consider also several biases in actual studies, including highly selected patients with normal cognitive function and no mood disorders at baseline, as

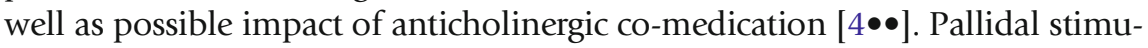
lation may induce Parkinsonism as bradykinesia, freezing, postural instability and micrographism $[8 \bullet \bullet]$. In most cases, compromise between Parkinson symptoms and dystonia can be achieved by adapting stimulation settings and diminution of total energy delivery.

Deep brain stimulation can also be considered in cranial and cervical dystonia after failure of botulinum toxin injections (level B) $[6 \bullet \bullet, 8 \bullet \bullet, 18]$. Pharmacoresistant cervical dystonia may show up to $70 \%$ improvement in Toronto Western Spasmodic Torticollis Rating Scale (TWSTRS) [15, 19]. Data regarding other forms of focal dystonia are limited to small series of mixed cases or individual reports, since focal dystonia are often sufficiently treatable with injection of botulinum toxin, showing encouraging but still highly variable results with mostly bilateral GPi-DBS $[4 \bullet \bullet]$. Some patients with severe writer's cramp have been successfully treated with unilateral DBS of the ventral oral (Vo) nucleus of the thalamus $[20,21]$. There is little experience with other targets for DBS in dystonia, with scant comparative data of DBS of subthalamic nucleus versus pallidal stimulation in patients with dystonia, and in case of coexisting tremor, thalamic targets $[8 \bullet \bullet]$.

Secondary dystonia as hemidystonia, cerebral palsy related dystonia as well as neurodegenerative forms (Wilson's disease, Lesch-Nyhan, panthotenate kinase-associated neurodegeneration (PKAN), etc.) are still being explored but 
seem to respond less well to pallidal DBS than primary dystonia. Application of DBS in dystonia secondary to brain injury is also strongly debated [22]. Patients with secondary dystonia often have complex movement disorders with combination of hyperkinetic and akinetic-rigid dystonia as well as pre-existing

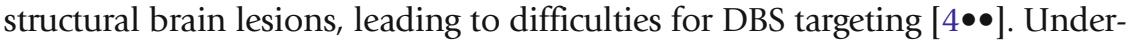
lying disease is often progressive, resulting at long term in DBS failure with re-

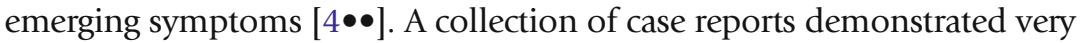
variable results in DBS treatment of dystonia symptoms in neurodegeneration with iron brain accumulation (PKAN) and its application in secondary dystonia in cerebral palsy; average improvement is around $20-25 \%$, bearing in mind the heterogeneity of this group of patients and the often unmodified persistence of

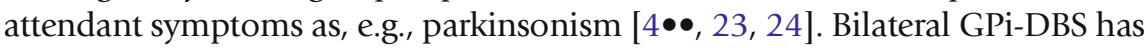
been performed in adults with dystonic-choreoathetotic cerebral palsy. Significant improvements in pain, functional disability and mental health-related quality of life have been reported [25]. In children, there is evidence to suggest better efficacy in early neurosurgical treatment before completion of motor development [26]. Considering DBS surgery in secondary dystonia as often less effective and with still limited experiences, indication should be carefully

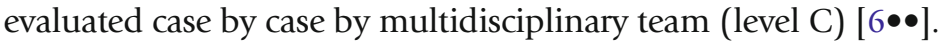

Tardive dyskinesia is a hyperkinetic movement disorder that appears with a delayed onset after prolonged use of dopamine receptor blocking agents, mainly neuroleptics. Clinical manifestation of tardive dyskinesia is very heterogenic, most commonly stereotypical involuntary movement affecting the lips and tongue, that may be associated to trunk and limb involvement. Less common is tardive dystonia (1$4 \%$ ), characterized by sustained muscle contraction leading to twisting repetitive movements and abnormal postures. Remission of dyskinetic symptoms has been reported in $14 \%$ of patients within a mean of 2.6 years after discontinuation of offending drugs and medical treatment remains often unsuccessful [5]. Tardive dystonia claims a special place among the tardive dyskinesia, because of the disability and the pain it may produce and its distinctive therapeutic options by the possibility of GPi-DBS, with similar benefit of DBS as reported in primary dystonia $[8 \bullet \bullet, 6 \bullet \bullet]$. In a follow-up from 3 to 76 months, more than $70 \%$ improvement of BFMDRS (Burke-Fahn-Marsden Dystonia Rating Scale) and extrapyramidal symptom rating scale (ESRS) has been reported [27]. It is likely that pathophysiology of tardive dystonia shares a common mechanism with primary dystonia, given their similar benefit of GPi-DBS $[8 \bullet \bullet, 6 \bullet \bullet, 28]$. Several studies reported improvement of extrapyramidal symptoms and dystonia within few hours (12-72 h) after starting GPi-high frequency stimulation, with improvement up to $80 \%$ of axial phasic dystonic movements and oro-buccal-lingual dyskinesia $[4 \bullet \bullet, 5,28]$. Benefit remains sustained on long-term follow-up of 8 years [28]. One study reports subthalamic nucleus DBS to be an effective therapy in refractory tardive dyskinesia; additional studies are needed to determine ideal stimulation target (GPi or subthalamic nucleus). Postoperative side effects after chronic stimulation are mostly mild and reversible by adjusting the stimulation parameters [5]. Even if no psychiatric or cognitive impairment after pallidal DBS in tardive dystonia reported, close psychiatric monitoring is recommended in this potentially vulnerable patient population [28]. 
Tremor is defined as a rhythmic and oscillatory movement of a body part with a relatively constant frequency and variable amplitude. Essential tremor has prevalence in general population ranging from 0.4 up to $6 \%$, with higher prevalence in people over 65 years of age [29], a 4-12 Hz postural and kinetic tremor concerning essentially upper extremities, less commonly also the lower extremities and the head. Essential tremor is improved in approximately $50 \%$ of the patients by medication [30]. About $25-55 \%$ of patients are refractory to medical treatment and are potential DBS candidates [29]. Target of DBS is the ventral intermediate nucleus (VIM) of thalamus [31 $\bullet$. Alternative target is the posterior subthalamic area (PSA; caudal zona incerta) [30]; these findings suggest that tremor control is best with stimulation of the cerebello-thalamic afferents, which are embedded in the subthalamic area [32]. Immediate and significant improvements in upper limb tremor are obtained with sustained effect in long-term follow-up up to 10 years [30, 33]. Head tremor may also improve in bilateral thalamic stimulation [34]. Outcome for voice- tremor is mixed and often only minimal symptom reduction is reported [34, 35]. DBS failure immediately postoperative is mostly due to suboptimal electrode placement. A gradual loss of efficacy of DBS may be due to phenomena of tolerance [16] and might be prevented by overnight withdrawal from stimulation $[36,37]$. Worsening of tremor over time may also be secondary to disease progression [38].

Efficacy of DBS in essential tremor and tremor of Parkinson's disease has led to apply the technique to rare secondary tremors. Tremor in MS patients has been the most studied. It has been estimated that tremor affects $26 \%$ MS patients, most commonly a postural or intention tremor, with $3 \%$ of patients suffering from a severe tremor [39, 40]. Effects of medication on MS tremor tend to be minor and short-lived [40,41]. The recent review of DBS in MS patients with tremor showed in all series a tremor reduction, with variable functional improvement [41]. Long-term follow-up are scare but point to vanishing benefit of DBS over time mainly in relation with disease progression [42]. Most common stimulation target was the VIM [41]. MS patients appeared to be less responsive to surgical intervention than those with essential tremor or secondary tremor in Parkinson's disease [43].

Holmes' tremor is characterized as a tremor present at rest, in antigravity postures and with action classically associated with midbrain lesions. It may have a levodopa-responsive component if lesion is involving the substantia nigra (pars compacta) [44]. Trials of application of DBS in Holme's tremor have shown to be more effective in GPi than thalamic DBS [44]. There are also few cases reported of thalamic DBS in posttraumatic tremor showing tremor improvement, although there is no follow-up greater than 1 year [44].

Further randomized controlled trials comparing efficacy and security of PSA and VIM stimulation are needed. DBS in treatment of essential tremor is relatively safe; most adverse events are mild, such as paraesthesia, headache and dysarthria and can mostly be treated by adjusting stimulation settings [45]. There is no overall change in cognitive functions described in VIM stimulation [30]. In order to reduce the risk related to the material and cost related to its regular replacement, alternative approaches as gamma knife $[46,47]$ and focused ultrasounds $[48,49]$ are under investigation with promising results. 


\section{DBS and Gilles de la Tourette syndrome}

Gilles de la Tourette syndrome (TS) is a disorder characterized by motor and phonic tics, often associated with other neuropsychiatric disorders, especially obsessive-compulsive disorders (OCD) and attention deficit hyperactivity disorder (ADHD). Onset is per definition before the age of 18 with a waxing and waning course of tics, with a peak of severity in early adolescence. Natural history of disease is favourable, characterized by subside of tic frequency and severity with increasing age. Treatment consists in comprehensive behavioural intervention for tics and medical treatment. In two third of patients, tics are completely regressive or only mildly persistent in early adulthood [50]. Only $5.1 \%$ of all TS patients present malignant TS with severe disability and impairment of social psychological and intellectual development despite optimized medical and behaviour therapy [51] representing potential DBS candidates. Studies for DBS in TS are scarce and mainly targeting the ventral thalamus (70 reported cases).

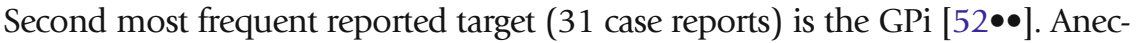
dotal reports exist also for the nucleus subthalamicus (STN), the external globus pallidus (GPe) and the anterior limb of capsula interna/nucleus accumbens. Because of small number of patients, lack of controlled studies and variability in outcome measures (including comorbidities of TS), there is currently almost no

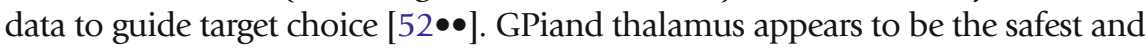
most effective targets [53].Few studies [54-57] reported higher benefice by bilateral GPi stimulation compared to bilateral thalamus stimulation. GPi-DBS seems to improve also behavioural comorbidities [54, 57, 58]. Considering particularities of this uncommon indication, involving vulnerable patients, DBS should only be offered after multidisciplinary evaluation to evaluate individual

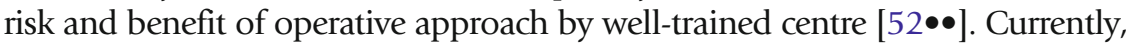
the 2014 revised guidelines for DBS candidates in TS require fulfilled TS diagnostic criteria (presence of more than 1 year of multiple motor and at least one phonic tic, with onset before age of 18 years) and persistence of severe motor or vocal tics despite adequate medical and behavioural treatment, with important social, psychological or intellectual impacts. Minimal age limit is fixed at 18 years, despite theoretically possible spontaneous tic remission, given the increasing evidence of a favourable risk-benefit ratio, as delaying surgery increases psychological, intellectual and social irreparable harm, as well as bodily harm through

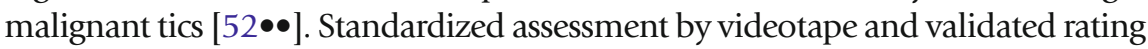
scale (e.g. Yale Global Tic Severity Rating Scale (YGTSS)) should be used for tic documentation, with a total YGTSS above 35/50 for at least 1 year. At least three different pharmacological trials-an alpha-adrenergic agonist, two dopamine antagonists (one typical and one atypical) and another drug class (topiramate,

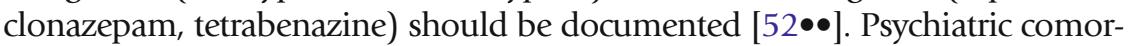
bidities should be carefully identified and adequately treated for at least 6 months before DBS surgery and should not be major source of functional impairment [59]. Exclusion criteria are structural lesions on brain MRI, substance abuse and risk of suicide. If deemed as good DBS candidate, a 30 to $50 \%$ or greater

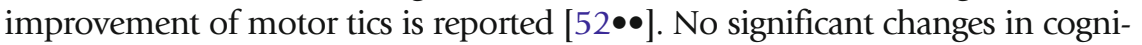
tion are reported after DBS application on the different targets. [57, 60]. Available data concerning effect of DBS on psychiatric comorbidities of TS patients are still limited, in part due to difficulties in their standardized evaluation, but are promising $[52 \bullet \bullet, 57,61,62]$. 
Ballism is a rare movement disorder consisting of non-voluntary ballistic motion of high amplitude in proximal limb, associated with a contralateral lesion of basal, most commonly due to stroke (ischemic/ haemorrhagic) or to hyperglycemia. Prognosis is generally favourable with complete resolution within 3 months [63]. DBS in hemiballism should only be considered in rare cases of pharamocoresistant ballism with symptoms persisting for more than 6-12 months [63]. Choreic movements, which are defined as random brief non-stereotyped involuntary movements affecting mostly distal limbs, have a large differential diagnosis, including hereditary neurodegenerative diseases, structural lesions of basal ganglia, autoimmune, infectious and metabolic/endocrine disorders, as well as drugs and toxins.

Improvement of hemiballism-hemichorea with thalamic stimulation (VIM) has been reported [64, 65] and more favourable regarding energy consumption compared to stimulation of GPi [65]. Several case reports of DBS in Huntington disease [66, 67] and neuroacanthocytosis [68] show benefit of stimulation in GPi, although resistance exists [69]. Benefit of stimulation of the thalamus in chorea in neuroacanthocytosis is also described [70]. In contrast with the improvement of chorea, other symptoms such as gait disturbances, dysarthria and hypotonia may develop or worsen [71]. Effects on cognitive functions is controversial, some reporting stability [67] while others showing cognitive decline [66]. Despite some promising postoperative results in chorea due to neurodegenerative disorders, long-term efficacy is probably limited by progression of underlying disease, as in secondary dystonia and tremor. Nevertheless, most surgical team currently prefer approach on GPi [68]; optimal target for stimulation remains debatable and long-term outcome is still uncertain, so that an individual multidisciplinary patient approach is mandatory.

Well-accepted indication in movement disorders and promising effects in other neurological and psychiatric disorders have lead to rapid expansion of DBS applications [71]. Precise mapping of functional territories of basal ganglia with their motor and non-motor functions will help to better understand behavioural and movement disorders and will permit better definition of the optimal therapeutical targets. In addition, further technical developments of hardware/electrodes will allow a better steering of the stimulation to optimize beneficial effects and reduce side effects, by directional electrodes [72] and close-loop intermittent stimulations. $[73,74]$. In order to reduce the risk related to open surgery, new lesional approaches (gamma knife, focused ultrasound) are starting to emerge [46-49]. There is need of multicentric international collaboration with defined protocols for these rare and specific DBS applications. In the meantime, DBS in most of hyperkinetic movement disorders (outside primary dystonia and essential tremor) should be restricted to expert centres. 


\section{Compliance with Ethics Guidelines}

\section{Conflict of Interest}

The authors declare that they have no competing interests.

\section{Human and Animal Rights and Informed Consent}

This article does not contain any studies with human or animal subjects performed by any of the authors.

\section{References and Recommended Reading}

Papers of particular interest, published recently, have been highlighted as:

- Of importance

$\bullet \quad$ Of major importance

1. Benabid AL et al. Long-term suppression of tremor by chronic stimulation of the ventral intermediate thalamic nucleus. Lancet. 1991;337(8738):403-6.

2. Schuurman PR et al. A comparison of continuous thalamic stimulation and thalamotomy for suppression of severe tremor. $\mathrm{N}$ Engl J Med. 2000;342(7):461-8.

3. Boviatsis EJ et al. Surgical and hardware complications of deep brain stimulation. A seven-year experience and review of the literature. Acta Neurochir (Wien). 2010;152:2053-62.

4.• Vidailhet M et al. Deep brain stimulation for dystonia. J Neurol Neurosurg Psychiatry. 2013;84(9):1029-42.

Excellent Review on DBS application in different dystonia forms.

5. Trottenberg T et al. Treatment of severe tardive dystonia with pallidal deep brain stimulation. Neurology. 2005;64(2):344-6.

6.• Albanese A et al. EFNS guidelines on diagnosis and treatment of primary dystonias. Eur J Neurol. 2011;18(1):5-18.

Actual European guidelines for dystonia management.

7. IM. Dystonia-new advances in classification, genetics, pathophysiology and treatment. Acta Neurol Scand Suppl. 2014 Apr;(198):13-9. Review.

8.• Moro E et al. What's new in surgical treatment for dystonia? Mov Disord. 2013 Jun 15;28(7):1013-20. Review.

This study provides a great overwiev on DBS treatment in dystonia.

9. Hendrix CM, Vitek JL. Toward a network model of dystonia. Ann N Y Acad Sci. 2012;1265:46-55.

10. Tisch $\mathrm{S}$ et al. Effect of electrode contact location on clinical efficacy of pallidal deep brain stimulation in primary generalised dystonia. J Neurol Neurosurg Psychiatry. 2007;78(12):1314-9.

11. Vidailhet $\mathrm{M}$ et al. Bilateral deep-brain stimulation of the globus pallidus in primary generalized dystonia. $\mathrm{N}$ Engl J Med. 2005;352(5):459-67.

12. Kupsch et al. Pallidal deep-brain stimulation in primary generalized or segmental dystonia. N Engl J Med. 2006;355(19):1978-90.

13. Cif $L$ et al. Treatment of dystonic syndromes by chronic electrical stimulation of the internal globus pallidus. J Neurosurg Sci. 2003;47(1):52-5.

14. Isaias IU et al. Deep brain stimulation for primary generalized dystonia: long-term outcomes. Arch Neurol. 2009;66(4):465-70.

15. Skogseid IM et al. Good long-term efficacy of pallidal stimulation in cervical dystonia: a prospective, observer-blinded study. Eur J Neurol. 2012;19(4):6105.

16. Cacciola F et al. Bilateral deep brain stimulation for cervical dystonia: long-term outcome in a series of 10 patients. Neurosurgery. 2010;67(4):957-63.

17. Anheim et al. Early stimulation of DYT1 primary generalized dystonia prevents from its secondary irreversible complications. Mov Disord. 2008;23(15):2261-3.

18. Kiss $\mathrm{ZH}$ et al. The Canadian multicentre study of deep brain stimulation for cervical dystonia. Brain. 2007;130(Pt 11):2879-86.

19. Walsh RA et al. Bilateral pallidal stimulation in cervical dystonia: blinded evidence of benefit beyond 5 years. Brain. 2013;136(Pt 3):761-9.

20. Goto $S$ et al. Thalamic Vo-complex vs pallidal deep brain stimulation for focal hand dystonia. Neurology. 2008;70(16 Pt 2):1500-1.

21. Cho CB et al. Thalamic deep brain stimulation for writer's cramp. J Korean Neurosurg Soc. 2009;46(1):52-5.

22. Katsakiori PF et al. Deep brain stimulation for secondary dystonia: results in 8 patients. Acta Neurochir (Wien). 2009 151(5):473-8. discussion 478.

23. Timmermann L et al. Dystonia in neurodegeneration with brain iron accumulation: outcome of bilateral pallidal stimulation. Brain. 2010;133(Pt 3):701-12.

24. Koy A et al. Effects of deep brain stimulation in dyskinetic cerebral palsy: a meta-analysis. Mov Disord. 2013;28(5):647-54. 
25. Vidailhet $M$ et al. French SPIDY-2 study group. Bilateral pallidal deep brain stimulation for the treatment of patients with dystonia-choreoathetosis cerebral palsy: a prospective pilot study. Lancet Neurol. 2009;8:709-17.

26. Lumsden DE et al. Proportion of life lived with dystonia inversely correlates with response to pallidal deep brain stimulation in both primary and secondary childhood dystonia. Dev Med Child Neurol. 2013;55(6):567-74.

27. Mentzel CL et al. Efficacy and safety of deep brain stimulation in patients with medication-induced tardive dyskinesia and/or dystonia: a systematic review. J Clin Psychiatry. 2012;73(11):1434-8.

28. Chang EF et al. Long-term benefit sustained after bilateral pallidal deep brain stimulation in patients with refractory tardive dystonia. Stereotact Funct Neurosurg. 2010;88(5):304-10.

29. Louis ED. Clinical practice. Essential tremor. N Engl J Med. 2001 Sep 20;345(12):887-91. Review.

30. Ondo W, Almaguer M, Jankovic J, Simpson RK. Thalamic deep brain stimulation: comparison between unilateral and bilateral placement. Arch Neurol. 2001;58:218-22.

31. Chopra A et al. Current clinical application of deepbrain stimulation for essential tremor. Neuropsychiatr Dis Treat. 2013;9:1859-65.

Good overview on DBS treatment in essential tremor.

32. Groppa S et al. Physiological and anatomical decomposition of subthalamic neurostimulation effects in essential tremor. Brain. 2014;137:109-21.

33. Sydow O, Thobois S, Alesch F, Speelman JD. Multicentre European study of thalamic stimulation in essential tremor: a six year follow up. J Neurol Neurosurg Psychiatry. 2003;74:1387-91.

34. Obwegeser AA et al. Thalamic stimulation for the treatment of midline tremors in essential tremor patients. Neurology. 2000;54(12):2342-4.

35. Sydow $\mathrm{O}$ et al. Multicentre European study of thalamic stimulation in essential tremor: a six year follow up. J Neurol Neurosurg Psychiatry. 2003;74(10):1387-91.

36. Hariz GM et al. Long-term effect of deep brain stimulation for essential tremor on activities of daily living and health-related quality of life. Acta Neurol Scand. 2008;118(6):387-94.

37. Barbe MT et al. Deep brain stimulation in the nucleus ventralis intermedius in patients with essential tremor: habituation of tremor suppression. J Neurol. 2011;258(3):434-9.

38. Favilla CG et al. Worsening essential tremor following deep brain stimulation: disease progression versus tolerance. Brain. 2012;135(Pt 5):1455-62.

39. Alusi $\mathrm{SH}$ et al. A study of tremor in multiple sclerosis. Brain J Neurol. 2001;124:720-30.

40. Roy HA, Aziz TZ. Deep brain stimulation and multiple sclerosis: therapeutic applications. Mult Scler Relat Disord. 2014;3(4):431-9.

41. Koch $\mathrm{M}$ et al. Tremor in multiple sclerosis. J Neurol. 2007;254:133-45.
42. Hassan et al. Surgical therapy for multiple sclerosis tremor: a 12-year follow-up study. Eur J Neurol. 2012;19(5):764-8.

43. Benabid AL et al. Chronic electrical stimulation of the ventralis intermedius nucleus of the thalamus as a treatment of movement disorders. J Neurosurg. 1996;84:203-14.

44. Starr PA. Deep brain stimulation for other tremors, myoclonus, and chorea. Handb Clin Neurol. 2013;116:209.15.

45. Flora ED et al. Deep brain stimulation for essential tremor: a systematic review. Mov Disord. 2010 Aug 15;25(11):1550-9. Review.

46. Ohye $\mathrm{C}$ et al. Gamma knife thalamotomy for Parkinson disease and essential tremor: a prospective multicenter study. Neurosurgery. 2012 Mar;70(3):52635. discussion 535-6.

47. Kooshkabadi A et al. Gamma knife thalamotomy for tremor in the magnetic resonance imaging era. J Neurosurg. 2013;118(4):713-8.

48. Lipsman $\mathrm{N}$ et al. MR-guided focused ultrasound thalamotomy for essential tremor: a proof-of-concept study. Lancet Neurol. 2013;12(5):462-8.

49. Wintermark $M$ et al. Imaging findings in MR imaging guided focused ultrasound treatment for patients with essential tremor. AJNR Am J Neuroradiol. 2014;35(5):891-6.

50. Bloch $\mathrm{MH}$ et al. Adulthood outcome of tic and obsessive-compulsive symptom severity in children with Tourette syndrome. Arch Pediatr Adolesc Med. 2006;160(1):65-9.

51. Cheung MY et al. Malignant Tourette syndrome. Mov Disord. 2007 Sep 15;22(12):1743-50. Review.

52.• Schrock LE et al. Tourette syndrome deep brain stimulation: a review and updated recommendations. Mov Disord. 2014 Dec 5.

This article reviewed most important literature on Tourette syndrome an summarize actual treatment guidelines for DBS application.

53. Piedad JC et al. What patients with gilles de la tourette syndrome should be treated with deep brain stimulation and what is the best target? Neurosurgery. 2012;71(1):173-92.

54. Welter ML et al. Internal pallidal and thalamic stimulation in patients with Tourette syndrome. Arch Neurol. 2008;65(7):952-7.

55. Martínez-Fernández R et al. Deep brain stimulation for Gilles de la Tourette syndrome: a case series targeting subregions of the globus pallidus internus. Mov Disord. 2012;26(10):1922-30.

56. Cannon E et al. Deep brain stimulation of anteromedial globus pallidus interna for severe Tourette's syndrome. Am J Psychiatry. 2012;169(8):860-6.

57. Saleh C et al. Deep brain stimulation of the globus pallidus internus and Gilles de la Tourette syndrome: toward multiple networks modulation. Surg Neurol Int. 2012;3 Suppl 2:S127-42. 
58. Viswanathan A et al. Deep brain stimulation for Tourette syndrome: target selection. Stereotact Funct Neurosurg. 2012;90(4):213-24.

59. Eddy $\mathrm{CM}$ et al. Clinical correlates of quality of life in Tourette syndrome. Mov Disord. 2011;26(4):735-8.

60. Ackermans L et al. Double-blind clinical trial of thalamic stimulation in patients with Tourette syndrome. Brain. 2011;134(Pt 3):832-44.

61. Zhang JG et al. Long-term outcome of globus pallidus internus deep brain stimulation in patients with Tourette syndrome. Mayo Clin Proc. 2014;89(11):1506-14.

62. Visser-Vandewalle V et al. Chronic bilateral thalamic stimulation, a new therapeutic approach in intractable Tourette Syndrome. J Neurosurg. 2003;99:1094-100.

63. Hawley JS, Weiner WJ. Hemiballismus: current concepts and review. Parkinsonism Relat Disord. 2012;18(2):125-9.

64. Nakano $\mathrm{N}$ et al. Successful long-term deep brain stimulation for hemichorea-hemiballism in a patient with diabetes. Case report. J Neurosurg. 2005;102(6):1137-41.

65. Capelle HH et al. Deep brain stimulation for treatment of hemichorea-hemiballism after craniopharyngioma resection: long-term follow-up. J Neurosurg. 2011;115(5):966-70.

66. Fasano A et al. GPi-DBS in Huntington's disease: results on motor function and cognition in a 72-year-old case. Mov Disord. 2008;23(9):1289-92.
67. Biolsi B et al. Long-term follow-up of Huntington disease treated by bilateral deep brain stimulation of the internal globus pallidus. J Neurosurg. 2008;109(1):130-2.

68. Edwards TC et al. Deep brain stimulation in the treatment of chorea. Mov Disord. 2012 Mar;27(3):357-63. Review.

69. Wihl G et al. Deep brain stimulation of the internal pallidum did not improve chorea in a patient with neuro-acanthocytosis. Mov Disord. 2001;16(3):572-5.

70. Burbaud $P$ et al. Improvement of severe trunk spasms by bilateral high-frequency stimulation of the motor thalamus in a patient with chorea-acanthocytosis. Mov Disord. 2002;17(1):204-7.

71. Kocabicak E et al. Current perspectives on deep brain stimulation for severe neurological and psychiatric disorders. Neuropsychiatr Dis Treat. 2015;11:1051-66.

72. Pollo et al. Directional deep brain stimulation: an intraoperative double-blind pilot study. Brain. 2014;137(Pt 7):2015-26.

73. Little $\mathrm{S}$ et al. Adaptive deep brain stimulation in advanced Parkinson disease. Ann Neurol. 2013;74(3):449-57.

74. Mahlknecht $P$ et al. Deep brain stimulation for movement disorders: update on recent discoveries and outlook on future developments. J Neurol. 2015 Jun 3. 